\title{
Pulsed Wave Doppler Echocardiographic Evaluation and Management of Atrial Fibrillation in Dogs with Dilated Cardiomyopathy
}

\author{
K. Basava Reddy ${ }^{1 *}$, C. Ansar Kamran ${ }^{1}$, H.A. Upendra ${ }^{1}$, Suguna Rao $^{2}$ and V. Mahesh ${ }^{3}$ \\ ${ }^{1}$ Department of Veterinary Medicine, Veterinary college, Hebbal, Benglore, Karnataka, INDIA \\ ${ }^{2}$ Department of Veterinary Pathology, Veterinary College, Benglore, Karnataka, INDIA \\ ${ }^{3}$ Department of Veterinary Surgery and Radiology, Veterinary college, Benglore, Karnataka, INDIA \\ *Corresponding author: KB Reddy; E-mail: drbasava2k10@gmail.com
}

Received: 29 May, 2021

Revised: 12 July, 2021

Accepted: 18 July, 2021

\begin{abstract}
Atrial fibrillation is usually a chronic supraventricular arrhythmia most commonly associated with canine dilated cardiomyopathy (DCM) and degenerative mitral valve disease in dogs. The management of atrial fibrillation is quite challenging with the control of rate and rhythm being the main strategies. In the present study, a total of 150 dogs were subjected to detailed clinical examination, physical examination, electrocardiography and echocardiography. Among 150 dogs, DCM was diagnosed in 58 dogs (38.66\%). Out of 58 DCM cases, 21 had atrial fibrillation. The predominant clinical signs noticed in DCM with atrial fibrillation were exercise intolerance, ascites, cardiac cachexia, coughing and syncope. The study of echocardiographic changes in dogs with atrial fibrillation may be helpful to predict the prognosis and survival time. All 21 dogs with DCM and atrial fibrillation were divided into two groups (Group I \& II) based on mitral E wave deceleration time (Edt) on Pulsed Wave Doppler. The mean values of Edt were $74.82 \pm 1.29 \mathrm{~ms}$ and $88.99 \pm 1.31 \mathrm{~ms}$ in Group I and Group II dogs respectively. All the dogs were treated with Tab. Pimobendan (0.25mg/kg b.wt, bid, PO), Tab. Digoxin (0.005 mg/kg b.wt, bid, PO), Tab. Enalapril (0.5 $\mathrm{mg} / \mathrm{kg}$ b.wt, bid, PO) and Tab. Furosemide ( $2 \mathrm{mg} / \mathrm{kg}$ b.wt, bid, PO). After 10 days of treatment, the echocardiography was repeated to measure the peak mitral $\mathrm{E}$ wave velocity and deceleration time. The study revealed a high degree of positive correlation between Edt and survival time, as indicated by the increase of survival time with every unit raise in Edt.
\end{abstract}

\section{HIGHLIGHTS}

(0 Atrial fibrillation was the most common arrhythmia in dogs with DCM.

0 Pimobendane with standard therapy improved the survival time in DCM dogs with AF.

(0 Mitral Edt was useful to predict prognosis and survival time in dogs with DCM and AF.

Keywords: Atrial fibrillation, Digoxin, Dogs, Pimobendan, Pulsed Wave Doppler

The etiology of Canine Dilated Cardiomyopathy (DCM) is unknown, although several factors have been proposed viz., hereditary, nutritional deficiency, toxins, viral infections. The atrial fibrillation is the most common arrhythmia in dogs with DCM due to atrial enlargement with high prevalence in large and giant breeds (NoszczykNowak et al., 2017). Clinical signs and physical examination findings of DCM with atrial fibrillation are exercise intolerance, syncope, ascites, tachycardia, systolic murmur, pleural and pericardial effusions (Ward et al., 2019). On physical examination, fast and irregularly irregular cardiac rhythm concomitant with marked pulse deficits indicate atrial fibrillation (Pedro et al., 2020). The ECG and echocardiography are the final diagnostic tools for the diagnosis of atrial fibrillation in dogs with dilated cardiomyopathy which is characterized by absence of visible $\mathrm{P}$ wave and irregular R-R intervals.

How to cite this article: Reddy, K.B., Kamran, C.A., Upendra, H.A. Rao, S. and Mahesh, V. (2021). Pulsed Wave Doppler Echocardiographic Evaluation and Management of Atrial Fibrillation in Dogs with Dilated Cardiomyopathy. J. Anim. Res., 11(04): 643-648.

Source of Support: None; Conflict of Interest: None 
The management of atrial fibrillation is quite challenging. The rate and rhythm control are the main strategies (Pedro et al., 2020). The dogs with preclinical atrial fibrillation treated with digoxin had a significantly shorter survival time (Vollmar et al., 2016). Pimobendane added to the standard therapies (i.e., furosemide, enalapril, digoxin) prolonged the survival time in Doberman pinschers (Fuentes et al., 2002). Addition of Carvedilol to the standard therapy improved echocardiographic indices of systolic function (FS and EF), reduced NT-proBNP levels but no significant improvement over standard therapy (Akhilesh et al., 2018). Myocardial dysfunction with atrial fibrillation reduces the survival time and quality of life in dogs with DCM. The successful management of atrial fibrillation in dogs with DCM may increase the survival time and improve the quality of life. The study of echocardiographic changes in dogs with atrial fibrillation may be helpful to predict the prognosis and survival time. Hence the present study was undertaken to study the pulsed wave doppler echocardiographic changes and survival time in dogs with atrial fibrillation secondary to dilated cardiomyopathy.

\section{MATERIALS AND METHODS}

Dogs presented to the Department of Veterinary Medicine, Veterinary College, Hebbal, Bangalore $(n=150)$ with clinical signs suggestive of cardiac insufficiency were screened for the DCM using the specially designed cardiology data sheet. They were subjected to detailed clinical examination, physical examination, electrocardiography and echocardiography. The ECG was recorded in right lateral recumbency as per the standard bipolar limb lead system described by Tilley and Smith (2008) using GE Healthcare-MAC-400 portable ECG machine. Standard transthoracic echocardiography was performed using LOGIC BOOK XP ultrasound system with a cardiac probe of 6 to 10 Megahertz $(\mathrm{MHz})$ phased array transducer. Furthermore, M-mode and Doppler echocardiography were carried out to confirm the DCM and to record the LV diastolic inflow from the left apical four chamber view by placing the sample volume at the level of mitral valve. The peak velocity and duration of early diastolic and late diastolic flow wave and ratio between the two peak velocities were measured by doppler echocardiography. The dogs that had Fractional shortening below twenty five percent, ejection fraction below fifty percent in M- mode echocardiogram and absence of discernible $\mathrm{p}$ waves in electrocardiography were selected for study. The mitral $\mathrm{E}$ wave velocity and deceleration time was measured at left apical four chamber view at the level of mitral valve. A total of 21 dogs diagnosed as DCM with atrial fibrillation belonged to different breeds and age groups (1-8 years) were divided in to two groups based on mitral E wave deceleration time on PWD. The dogs with mitral E wave deceleration time $<80$ milli sec were included in Group I and dogs with mitral E wave deceleration time $>80$ milli sec were included in Group II. All the dogs were treated with Tab. Pimobendan ( $0.25 \mathrm{mg} / \mathrm{kg}$ b.wt., bid, PO), Tab. Digoxin (0.005 mg/kg b.wt., bid, PO), Tab. Enalapril $(0.5 \mathrm{mg} / \mathrm{kg}$ b.wt, bid, PO) and Tab. furosemide ( $2 \mathrm{mg} / \mathrm{kg}$ b.wt, bid, PO). The echocardiography was repeated after 10 days of treatment to measure the mitral diastolic inflow velocity and deceleration time (Edt). The survival time (days) was calculated based on date of cardiac related death or euthanasia related with worsening of congestive heart failure (CHF). Least squares means of deceleration time (milli sec), survival time (days) and heart rates (bpm) and correlations between them were studied by using SPSS ver 20.0 software.

\section{RESULTS AND DISCUSSION}

In the present study, a total of 150 dogs were subjected to detailed clinical, physical examination, electrocardiography and echocardiography. Among 150 dogs screened, 58 dogs (38.66\%) were diagnosed as DCM and out of 58 DCM cases 21 had atrial fibrillation. The predominant clinical signs noticed in DCM dogs with atrial fibrillation on the day of presentation were exercise intolerance, lethargy, ascites, cardiac cachexia, laboured breathing, coughing and syncope. Similar clinical findings were also reported by the earlier authors (Jeyaraja et al., 2015; Puri et al., 2015; Baisan et al., 2016; Thangapandiyan et al., 2016; Harmon et al., 2017). These clinical signs might be due to atrial fibrillation alone or in combination with congestive heart failure. Syncope could be due to sudden loss of atrial contribution to ventricular filling (Pedro et al., 2020). Atrial fibrillation was the most common arrhythmia diagnosed in dogs with DCM that could be due to atrial stretching (Borgarelli et al., 2006). Severe left atrial dilatation in DCM dogs was the reason behind the increased incidence of AF in dogs with DCM (Jeyaraja et al., 2015). The peak mitral inflow E wave and 
A wave velocities in healthy dogs were $0.747 \pm 0.117 \mathrm{~m} / \mathrm{s}$ and $0.487 \pm 0.062 \mathrm{~m} / \mathrm{s}$ respectively (Pereira, 2009). In the present study among 21 dogs diagnosed as DCM with atrial fibrillation by ECG and Echocardiography, 16 (76.19\%) had prominent mitral E wave (peak early diastolic filling wave) with increased velocity $(>0.8 \mathrm{~m} / \mathrm{s})$ and the absence of A wave (peak late diastolic filling wave) on PWD (Fig. 1) which could be due to increased left atrial pressure, restrictive flow pattern and loss of ventricular compliance. The remaining five dogs $(23.80 \%)$ had normal mitral $\mathrm{E}$ wave velocity $(<0.8 \mathrm{~m} / \mathrm{s})$ with lower A wave velocity which might be due to mild atrial contraction that causes little additional ventricular filling during late diastole. The mitral diastolic inflow $\mathrm{E}$ wave deceleration time in healthy dogs was $88.7 \pm 9.2 \mathrm{~ms}$ and can be used to assess the diastolic function in dogs (Pereira, 2009). In the present study, the mean mitral valve $\mathrm{E}$ wave deceleration time (Edt) was $74.82 \pm 1.29 \mathrm{~ms}$ and $88.99 \pm 1.31 \mathrm{~ms}$ in Group I and Group II respectively. The reduced Edt might be due

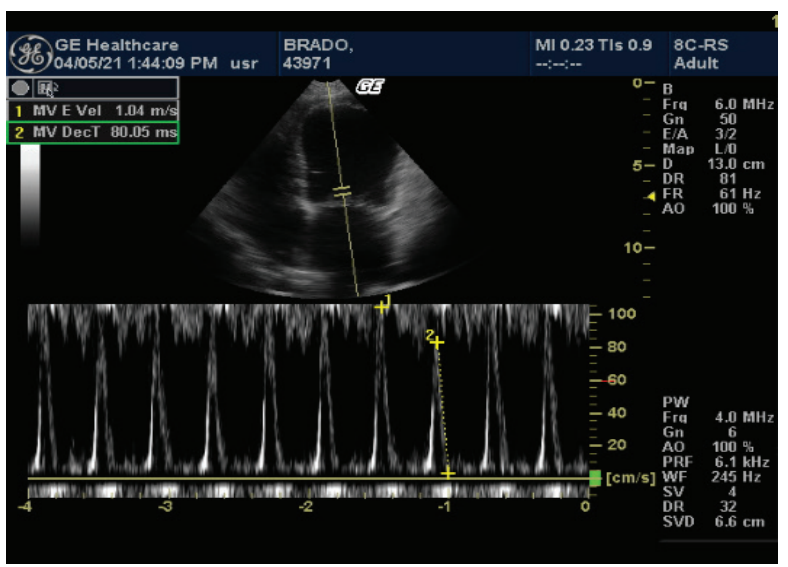

Fig. 1: PWD in a dog with dilated cardiomyopathy showing the prominent $\mathrm{E}$ wave (Edt:80.05ms) and the absence of A wave because of atrial fibrillation to increased left atrial pressure with chamber dilatation and left ventricular diastolic dysfunction. All the $21 \mathrm{dogs}$ with DCM and AF were treated with Tab. Pimobendan (0.25 mg/kg b.wt., bid, PO), Tab. Digoxin (0.005 mg/ $\mathrm{kg}$ b.wt., bid, PO) Tab. Enalapril (0.5 mg/kg b.wt, BID, PO) and Tab. furosemide ( $2 \mathrm{mg} / \mathrm{kg}$ b.wt., BID, PO) and reviewed after 10 days of treatment. Pimobendan can be combined with standard cardiovascular drugs (Diuretics, Angiotensin Converting Enzyme (ACE) inhibitors or digoxin) with no apparent adverse effects and improved the cardiac function in heart failure dogs (Boothe, 2012). After treatment, a total of seven dogs in Group I and twelve dogs in Group II showed improvement in condition with respect to exercise intolerance, reduced abdominal distension and improved breathing. Despite the treatment, the remaining two dogs in Group I died due to advanced stage of congestive heart failure and atrial fibrillation. In group II, two out of 12 dogs reported sinus rhythm after 10 days of treatment (Fig. 3, 4). A total of seven dogs in group

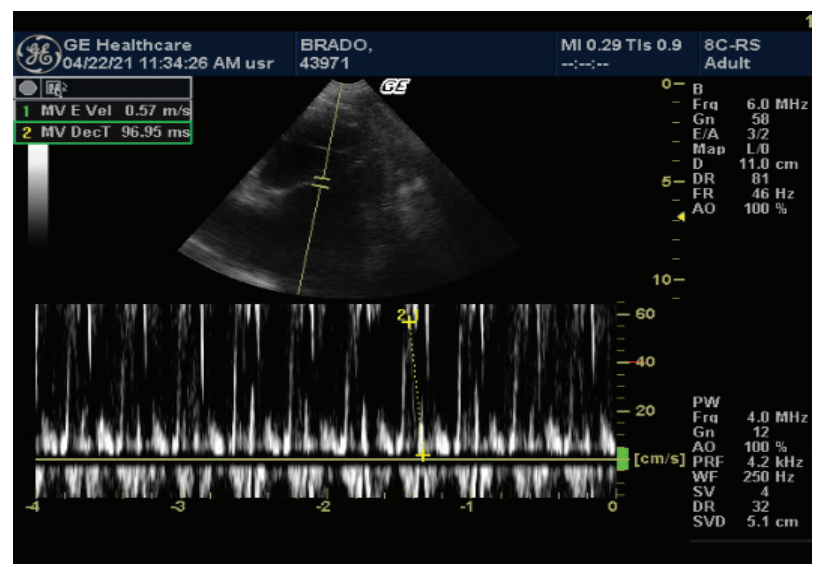

Fig. 2: PWD in the same dog of fig (1) after treatment showing the increased $\mathrm{E}$ wave deceleration time $(96.95 \mathrm{~ms})$ and a visible short A wave

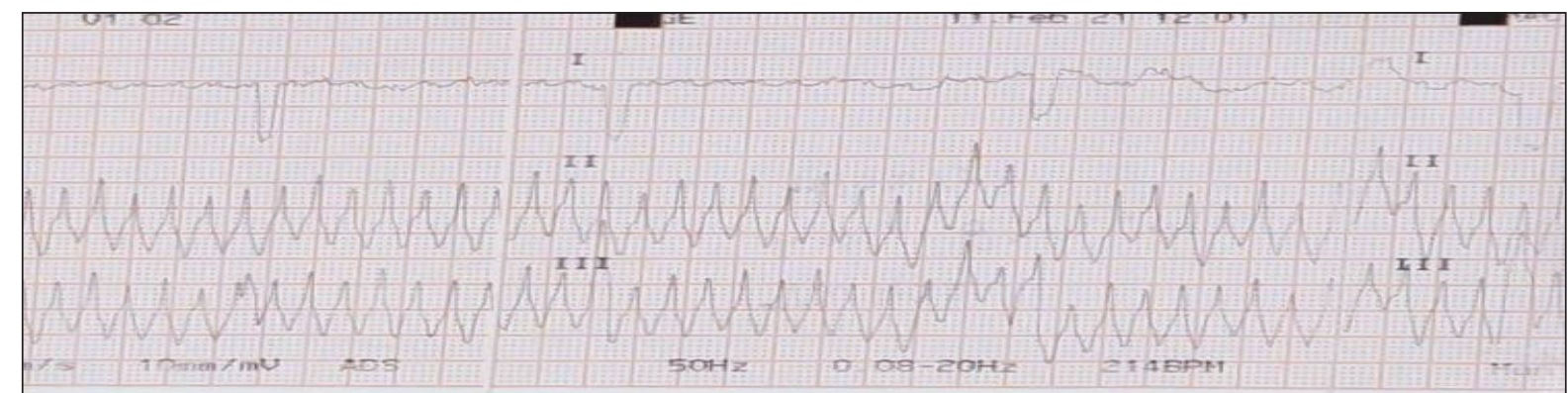

Fig. 3: Atrial fibrillation in a dog with fast ventricular response rate. The $\mathrm{P}$ wave is absent and The QRS complexes duration is prolonged. Paper speed $25 \mathrm{~mm} / \mathrm{s}$ 


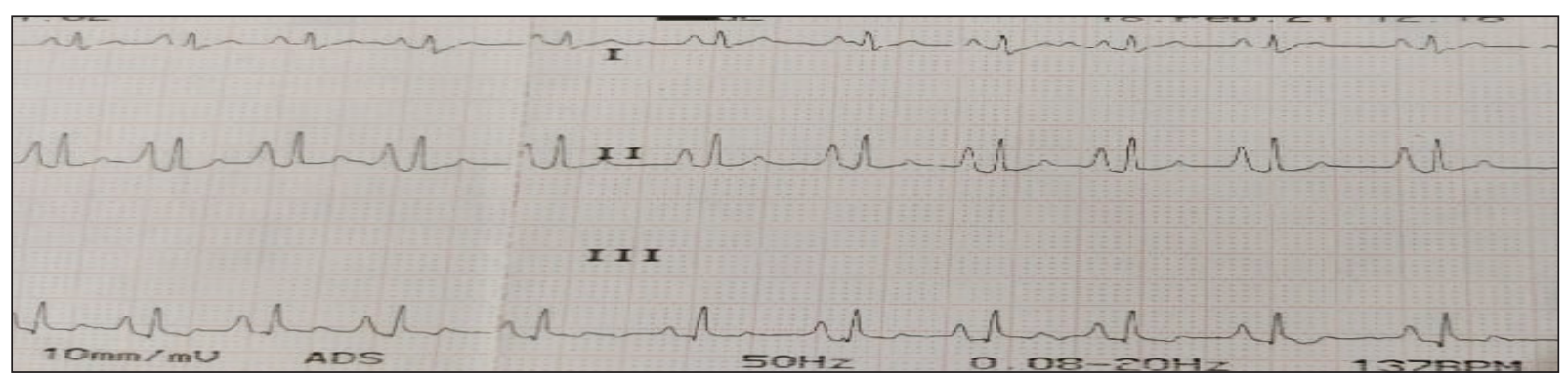

Fig. 4: ECG in the same dog of fig (1) after treatment. Sinus rhythem is reported by the presence of P wave and irregular R-R intervals. Paper speed $25 \mathrm{~mm} / \mathrm{s}$

I and 10 dogs in group II were managed with adequate heart rate $(<160 \mathrm{bpm})$ control with the above treatment protocol. In Group I, the mean mitral Edt was increased from $74.82 \pm 1.29 \mathrm{~ms}$ to $76.40 \pm 2.24 \mathrm{~ms}$ after treatment and there was no significant difference between them which might be due to advanced stage of CHF and atrial fibrillation. While in group II the mean mitral Edt was increased from $88.99 \pm 1.31 \mathrm{~ms}$ to $91.06 \pm 1.53 \mathrm{~ms}$ which is statistically significant $(\mathrm{P} \leq 0.05)$ and this might be due to retained left ventricular diastolic filling capacity after treatment.

The estimated marginal means along with standard errors for deceleration time, survival time, heart rates were presented in tables 1 and 2 respectively.

Table 1: Least square means of Deceleration time (ms) before and after treatments and survival time (days) in group I and II dogs

\begin{tabular}{lllll}
\hline Animals & $\begin{array}{l}\text { Sample Edt Before } \\
\text { size (n) }\end{array}$ & $\begin{array}{l}\text { Edt after } \\
\text { treatment }(\mathbf{m s})\end{array}$ & $\begin{array}{l}\text { Survival } \\
\text { treatment }\end{array}$ & $\begin{array}{l}\text { time (days) } \\
\text { (ms) }\end{array}$ \\
\hline $\begin{array}{l}\text { Group I } \\
(\mathrm{DT}<80)\end{array}$ & 9 & $74.82 \pm 1.29^{\mathrm{NS}}$ & $76.40 \pm 2.24^{\mathrm{NS}}$ & $164.67 \pm$ \\
& & & 31.32 \\
$\begin{array}{l}\text { Group II } \\
(\mathrm{DT}>80)\end{array}$ & 12 & $88.99 \pm 1.31^{\mathrm{b}}$ & $91.06 \pm 1.53^{\mathrm{a}}$ & $286 \pm 15.75$ \\
\hline
\end{tabular}

Note: Different superscripts in lower case indicates significant difference at $5 \%$ level of significance, NS: Non significant.

Correlation between heart rate, deceleration time and survival time are calculated and are shown in table 3 . There is a high degree of positive correlation between Edt and survival time which indicates that with every unit raise in Edt, there will be an increase in survival time.
Table 2: Least square means of Heart rate (bpm) before and after treatment and survival time (days) in group I and II dogs

\begin{tabular}{|c|c|c|c|c|}
\hline Animals & $\begin{array}{l}\text { Sample } \\
\text { size (n) }\end{array}$ & $\begin{array}{l}\text { HR before } \\
\text { treatment } \\
(\mathrm{bpm})\end{array}$ & $\begin{array}{l}\text { HR after } \\
\text { treatment } \\
(\text { bpm) }\end{array}$ & $\begin{array}{l}\text { Survival } \\
\text { time (days) }\end{array}$ \\
\hline $\begin{array}{l}\text { Group I } \\
(\mathrm{DT}<80)\end{array}$ & 9 & $182 \pm 18.01^{\mathrm{a}}$ & $\begin{array}{l}135.22 \pm \\
11.39^{\mathrm{b}}\end{array}$ & $\begin{array}{l}164.67 \pm \\
31.32\end{array}$ \\
\hline $\begin{array}{l}\text { Group II } \\
(\mathrm{DT}>80)\end{array}$ & 12 & $171.5 \pm 8.74^{\mathrm{A}}$ & $\begin{array}{l}134.08 \pm \\
5.27^{\mathrm{B}}\end{array}$ & $\begin{array}{l}286 \pm \\
15.75\end{array}$ \\
\hline
\end{tabular}

Note: Different superscripts in lower case indicates significant difference at $5 \%$ level of significance and Different superscripts in upper case indicates significant difference at $1 \%$ level of significance.

The correlation between Edt with HR (-0.09 and -0.07) and HR with survival time (-0.01 and 0.04$)$ was found to be negative for both the groups respectively which indicates that with increase in heart rate, there will be a decrease in survival time and Edt.

Table 3: Correlations among Deceleration time, Heart rate and Survival time in Group I and II dogs

\begin{tabular}{lllll}
\hline & \multicolumn{2}{c}{ Group I } & \multicolumn{2}{c}{ Group } \\
\hline & Edt before & HR before & Edt before & HR before \\
\hline $\begin{array}{l}\text { HR before } \\
\text { Survival }\end{array}$ & -0.09607 & 1 & -0.072 & 1 \\
time & $0.90^{* *}$ & -0.0167 & 0.4136 & 0.0493 \\
\hline
\end{tabular}

Note: ** indicates $\mathrm{P} \geq 0.01$.

In the present study mean survival time (MST) was reduced in group I (164.67 \pm 31.32 days) when compared with group II $(286 \pm 15.75$ days $)$. The results of the present study are similar with the findings of Chetboul, 2016 who reported that short deceleration time of $\mathrm{E}$ wave $(<80 \mathrm{~ms})$ has negative prognostic value in the presence of 
atrial fibrillation. The relationship between Edt, HR and survival time are presented using bubble diagram in Fig. 5 and 6 respectively for group I and II dogs separately.

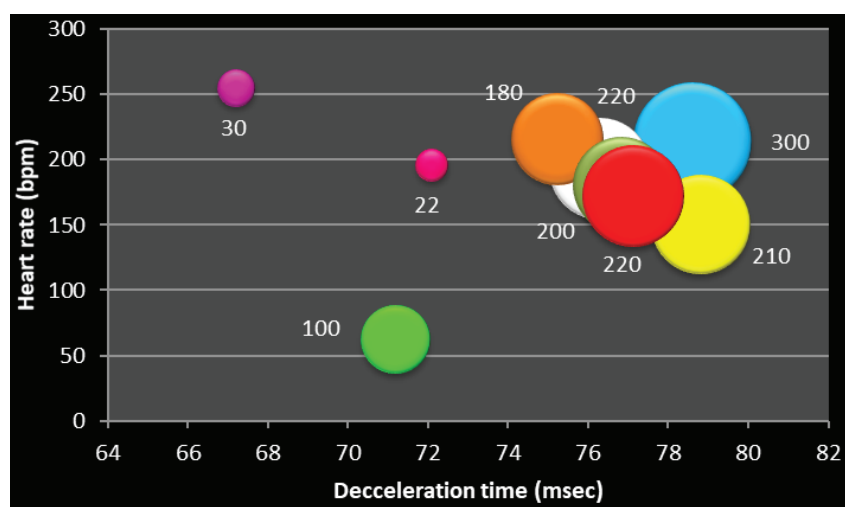

Fig. 5: Relationship between deceleration time (milli sec), heart rate (bpm) and survival time (days) in Group I animals

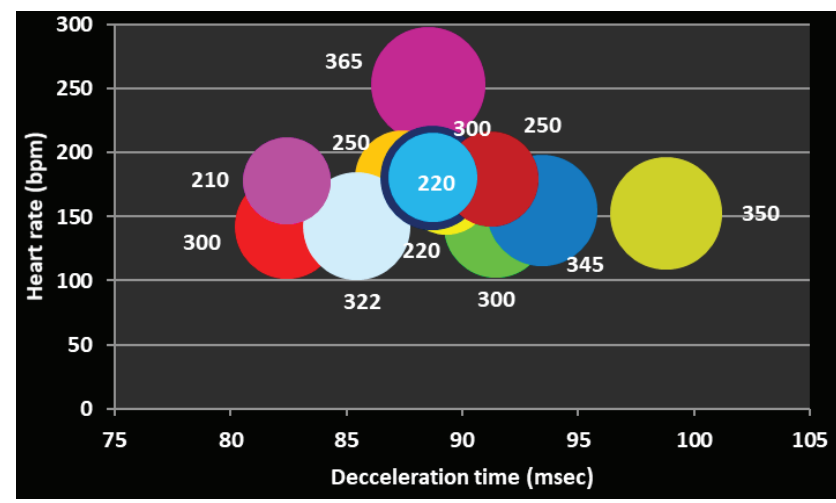

Fig. 6: Relationship between deceleration time (milli sec), heart rate (bpm) and survival time (days) in Group II animals

\section{CONCLUSION}

The atrial fibrillation was the most common arrhythmia in dogs with dilated cardiomyopathy which can be managed and improve the survival time with antiarrhythmic and positive inotropic medications. The addition of pimobendane to the standard therapy (digoxin, diuretics, ACE inhibitors) improved the survival time and quality of life in dogs with DCM and atrial fibrillation. The absence of mitral A wave (peak late diastolic filling wave) and decreased Edt $(<80 \mathrm{~ms})$ had negative prognostic value and decreased the survival time due to loss of ventricular compliance.

\section{REFERENCES}

Akhilesh, K., Sahadeb, D., Abhishek, C.S. and Sumit, M. 2018. Evaluation of add on effect of carvedilol on standard protocol of digoxin, frusemide and benazepril in the management of dilated cardiomyopathy in dogs. Indian J. Anim. Res., 52(4): 583-590.

Baisan, R.A., Birsan, O. and Vulpe, V. 2016. Electrocardiographic changes in chronic valvulardisease and dilated cardiomyopathy in dog. Hum. Vet. Med., 8(2): 98-102.

Boothe, D.M. 2012. Small Animal Clinical Pharmacology and Therapeutics: Therapy of cardiovascular diseases. Edn.2, Elsevier Saunders, Missouri, pp. 469-567.

Borgarelli, M., Santelli, R.A., Chiavegato, D., Agnolo, G.D., Zanatta, R., Mannelli, A. and Tarducci, A. 2006. Prognostic indicators for dogs with dilatedcardiomyopathy. J. Vet. Intern. Med., 20: 104-110.

Chetboul, V. 2016. Dilated cardiomyopathy and other cardiomyopathies in dogs - Clinical echocardiography of the dog and cat: Elsevier, Missouri, pp. 182-194.

Fuentes, V.L., Corcoran, B. and French, A. 2002. A doubleblinded, randomized placebo-controlled study of pimobendane in dogs with dilated cardiomyopathy. $J$. Vet. Intern. Med., 16(3): 255-261.

Harmon, M.W., Leach, S.B. and Lamb, K.E. 2017. Dilated cardiomyopathy in standard schnauzers: retrospective study of 15 cases. J. Am. Anim. Hosp. Assoc., 53(1): 38-44.

Jeyaraja, K., Arun, A., Hamsa Yamini, S., Sesh, P.S. and Nambi, A.P. 2015. Diagnostic evaluation of dilated cardiomyopathy in labrador retrievers. Int. J. Adv. Res., 3(12): 628-655.

Noszczyk-Nowak. A., Michalek, M., Kaluza, E., Cepiel, A. and Paslawska, U. 2017. Prevalence of arrhythmias in dogs examined between 2008 and 2014. J. Vet. Res., 61: 103-110.

Pedro, B., Fontes-Sousa, A.P. and Gelzer, A.R. 2020. Diagnosis and management of canine atrial fibrillation. Vet. J., 265: $1-38$.

Pereira, G.G., Petrus, L.C., Santos, A.L.F., Yamaki, F.L. and Larsson, M.A. 2009. Evaluation of left ventricular diastolic echocardiographic parameters in healthy dogs by pulsedwave Doppler. Pesq. Vet. Bras., 29(4): 291-294.

Puri, D., Bhat, S.A., Thakur, A. and Sudhan, N.A. 2015. Dilated cardiomyopathy in geriatric dogs. Ind. J. Canine Pract., 7(2): 132.

Thangapandiyan, M., Mohanapriya, T., Balachandran, C., Jeyaraja, K. and Arulanandam, K. 2016. Study on pathomorphological changes in 21 cases of canine dilated cardiomyopathy. Int. J. Sci Environ. Technol., 5(6): 46124617. 
Vollmar A.C. and Fox P.R. 2016. Long-term outcome of Irish wolfhound dogs with preclinical cardiomyopathy, atrial fibrillation, or both treated with pimobendane, benazepril hydrochloride, or methyldigoxine monotherapy. J. Vet. Intern. Med., 30: 553-559.
Ward, J., Ware, W. and Viall, A. 2019. Association between atrial fibrillation and right-sided manifestations of congestive heart failure in dogs with degenerative mitral valve disease or dilated cardiomyopathy. J. Vet. Cardiol., 21: 18-27. 